\title{
Myofibrosarcoma Mimicking a Vascular Thrombosis: A Case Report
}

\section{Introduction}

Myofibrosarcoma is a very rare subtype of sarcoma derived from myofibroblasts. This tumor affects mainly adults, with a slight male predominance (Fisher C. Virchows Arch. 2004; 445: 215-223). It has a wide anatomical distribution but shows a predilection for the head and neck, especially the oral cavity (Mentzel T. AmJ Surg Pathol. 1998; 22: 12281238). Myofibrosarcoma is a low-grade sarcoma and has a low metastasis risk but a high recurrence rate. The purpose of this study is to report a case of myofibrosarcoma of the neck presenting with imaging findings suggestive of a vascular thrombosis.

\section{Case Report}

A 57-year-old female patient with a past history of T4aN1 bM0, stage IV, papillary carcinoma of the thyroid had undergone thyroidectomy with excision of the overlying strap muscle, ipsilateral esophageal musculature, and left recurrent laryngeal nerve; partial left laryngectomy; left selective neck dissection; and left pectoralis major muscle flap reconstruction followed by postoperative radiation therapy. Seventeen months after radiation, the patient presented with a new left inferior neck mass and no symptoms.

\section{Imaging}

A computed tomography scan revealed a tubular mass in the left inferior neck and shoulder region ( $\bullet$ Fig. $\mathbf{1}$ ) and was believed to represent a "prominent vessel". Ultrasonography showed a hypoechoic, avascular tubular structure in the posterior neck, thought to represent a thrombosed vessel. Repeat ultrasonography 6 weeks later showed an increase in the size of the tubular structure, now demonstrating vascular flow ( $\bullet$ Fig. 2). Treatment planning MRI showed a 5.5-cm ovoid, T2-hyperintense, enhancing tumor deep in the trapezius muscle without invasion.

\section{Pretreatment histology and immunohistochemistry}

Upon core biopsy, the tumor was found to be composed of spindle cells with a myxoid background and extension into the adjacent soft tissues. The tumor cells were neg- ative for keratins (which are most often seen in epithelial-derived neoplasms), PAX8 (a marker common in thyroid carcinoma that is retained in $\sim 50 \%$ of anaplastic carcinomas), and other mesenchymal-specific markers including desmin, SMA, and S100. The cells retained $\mathrm{Rb}$ (retinoblastoma) staining (often lost in spindle cell/pleomorphic lipomas). CD34 staining highlighted predominately background vasculature (helping to exclude solitary fibrous tumor). In an image analysis performed using Aperio ImageScope v12, the Ki67 expression level was 28.3 \% (of a total of 7849 nuclei). Overall, the immunohistochemical features supported a mesenchymal process most consistent with grade 1 to 2 myofibrosarcoma (low to intermediate grade) ( $>$ Fig. 3,4 ).

\section{Treatment}

The patient was treated with preoperative radiation at a total dose of 5000 cGy in 200cGy daily fractions using intensity-modulated proton therapy, followed by surgical excision. During the operation, the tumor was found to have an intact capsule with negative margins and no lymph node involvement or bone invasion. Gross total resection ( $>$ Fig. 5) included left extended selective neck dissection level $\mathrm{Va}$ and $\mathrm{Vb}$ with resection of a portion of the trapezius (performed superiorly), the levator scapulae, and the rhomboid minor. Adjuvant chemotherapy included 6 cycles of Adriamycin $75 \mathrm{mg} / \mathrm{m}^{2}$ and ifosfamide $10 \mathrm{mg} / \mathrm{m}^{2}$.

\section{Final pathology}

Gross evaluation of the tumor revealed a lobulated, tan-pink soft tissue mass (> Fig. 5) with a fibrotic capsule likely formed after radiation therapy. Final pathologic evaluation showed progression to a high-grade lesion or undifferentiated pleomorphic sarcoma (• Fig. 6).

Following chemotherapy, the patient has been followed under surveillance for 4 months to date.

\section{Discussion}

Myofibrosarcomas are rare neoplasms, with only a few cases described in the literature, the first by Eyden et al. in 1992 (Eyden BP. J

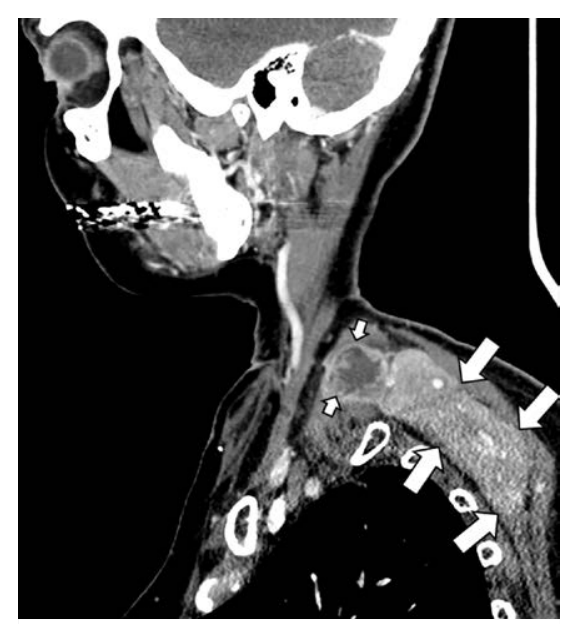

- Fig. 1 Sagittal computed tomography with contrast shows the tubular, enhancing mass in the inferior neck and shoulder (large arrows). Note the superior cystic component (small arrows).

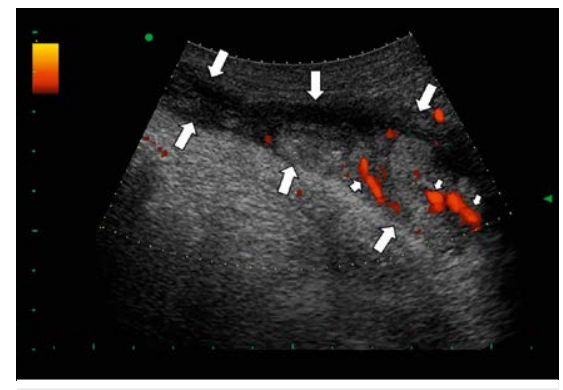

> Fig. 2 Repeat Doppler ultrasonography demonstrates the tubular mass (large arrows) in the left inferior neck and shoulder with increased vascular flow (small arrows).

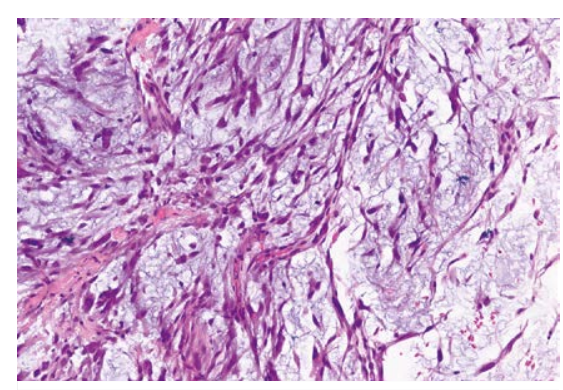

-Fig. 3 A hematoxylin and eosinstained tissue section from the biopsy shows spindle tumor cells with a myxoid background. No normal tissue is present. $(200 \times)$. 


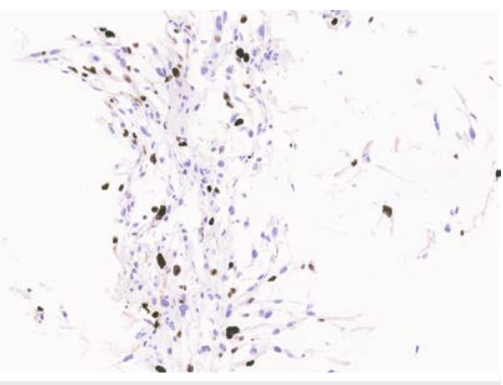

> Fig. 4 Enlarged, spindled tumor cell nuclei were positive for ki67, a proliferation marker by immunohistochemical analysis. $(200 \times)$.

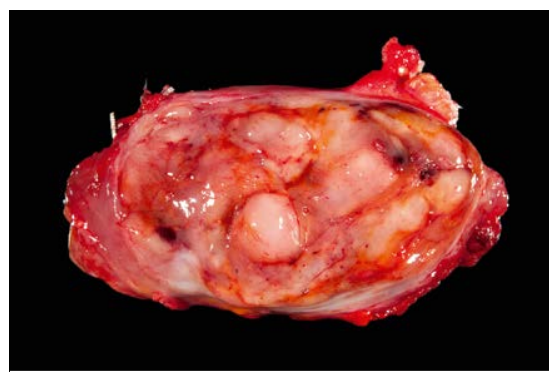

- Fig. 5 Photograph of the resected specimen shows an encapsulated tan-pink soft tissue tumor.

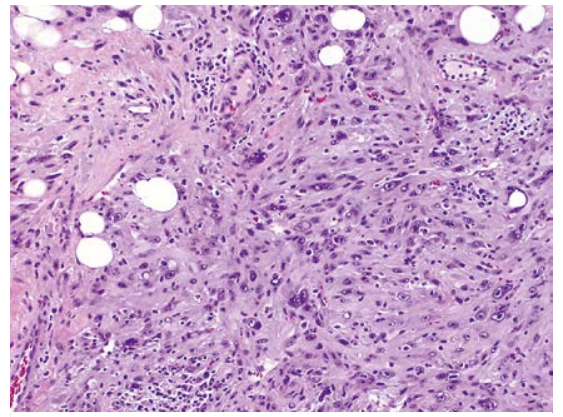

- Fig. 6 A hematoxylin and eosin-stained tissue section from the surgical resection (after radiation therapy) shows enlarged, pleomorphic tumor cells invading into fat. The increased cellularity and histologic features indicate an undifferentiated pleomorphic sarcoma. Scattered inflammatory cells with small hyperchromatic nuclei were noted in the background. $200 \times$

Submicrosc Cytol Pathol. 1992; 24:307-313). These are infiltrative tumors arising in the soft tissue or bone with a predilection for the head and neck area followed by the extremities and trunk (Fisher C. Virchows Arch. 2004; 445:215-223). Myofibrosarcomas are derived from myofibroblasts, modified fibroblasts found in some normal tissue but mainly in inflammatory and reparative granulation tissue (Eyden, BP. J Submicrosc Cytol Pathol. 1994; 26: 347-355).

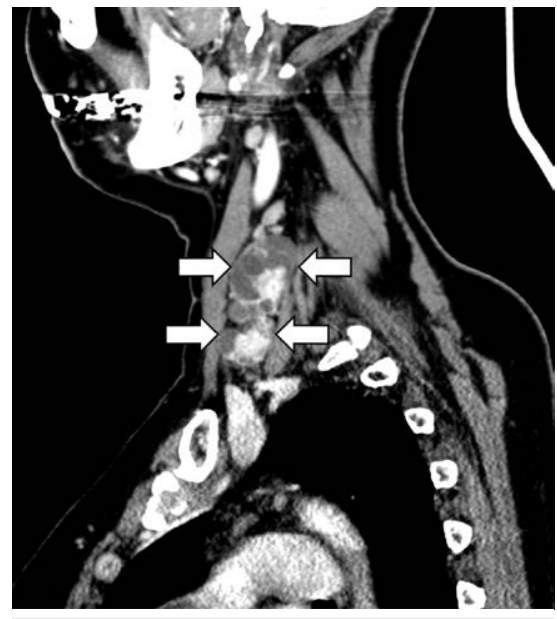

- Fig. 7 Contrast-enhanced CT scan done 17 months earlier, prior to radiation therapy, shows recurrent nodes in the left lower neck. The site of the myofibrosarcoma was unremarkable.

In total, 68 cases of head and neck myofibrosarcomas have been reported. The mean age of these patients was 42 years, with a slight male predominance (1.4:1). Most patients presented with a slowly growing painless mass. Radiologic findings have been described in 29 of these patients. All of the tumors presented as solid, soft-tissue masses, of which 8 arose from bone. Of the 21 lesions that arose from soft tissue, 14 lesions (67\%) showed bone erosion. Most of the tumors showed high signal intensity on T2-weighted magnetic resonance imaging sequences and low intensity on T1-weighted magnetic resonance imaging with contrast enhancement. Metastasis has been found in only two cases of myofibrosarcoma (3.1\%). Treatment of myofibrosarcoma has been described in 54 patients. Surgery was the mainstay of therapy in 52 of these 54 patients (96\%); the tumor was unresectable in two patients. Surgery was supplemented by radiation only in 10 patients (19\%), chemotherapy only in 2 patients (4\%), and both radiation and chemotherapy in 2 patients (4\%). Only 2 of 54 patients (4\%) were treated with radiation and chemotherapy alone, without surgery. Recurrence was reported in 23 of 52 cases (36\%), but long-term results were generally good: only 7 of the patients (10\%) died from the disease. As myofibrosarcoma has a low risk of metastasis but a high recurrence rate, complete surgical excision as performed in the present case offers the best chance of cure.
Mentzel et al. established the histologic criteria for the diagnosis of myofibrosarcoma in 1998. Histologically, myofibrosarcomas are composed primarily of spindle-shaped cells arranged in fascicles. The tumor cells have small to moderate amounts of ill-defined, mildly eosinophilic cytoplasm and fusiform nuclei, mild nuclear pleomorphism, and a low mitotic rate. In our case, the morphologic features align with those previously described, including elongated spindle-shaped cells with mild atypia and only an occasional mitotic figure. Immunohistochemically, these tumors are positive for vimentin, SMA, muscle-specific actin, calponin, and fibronectin; rarely positive for desmin; and negative for laminin, type IV collagen, S100, EMA, cytokeratin, and CD34 (Yamada T. Int J Oral Sci. 2012; 4: 170-173). While immunohistochemical studies can facilitate tumor classification and lineage, the absence of some of these markers in this case does not preclude this classification.

Post-radiation sarcoma is a well-known long-term complication of radiation therapy, with an incidence of $0.03 \%$ to $0.3 \%$ (31). The mean latency period before diagnosis is 15 years, with a range of 2 to 65 years in one study (Murray EM. Int J Radiat Oncol Biol Phys. 1999; 45: 951-961) and 3.5 to 65 years in another (Sheppard DG. Clin Radiol. 2001; 56: 22-29). A large portion of the tumor in our patient was within the previous high-dose radiation field for the papillary thyroid carcinoma recurrent nodes in the left lower neck ( $\triangleright$ Fig. 7). Although it appeared only 17 months after irradiation, this could still represent a case of post-radiation sarcoma.

Of special importance is the radiologic presentation of the myofibrosarcoma in our patient. The lesion was described as a "prominent vessel" in the initial computed tomography and was thought to be a thrombosed vein on ultrasonographic evaluation of the neck. On repeat ultrasonography, vascular flow was noted in the mass. Thrombosed vessels show no flow on duplex ultrasonography (Alpern MB. Radiology. 1987; 162: 53-56). The presence of arterial flow in our case prompted the acquisition of tissue samples. Arterial flow should be considered atypical or suspicious in cases of suspected thrombosis with vascular flow. 
Authors

Ryan Bou Said ${ }^{1}$, Michelle D. Williams², Beth S. Edeiken-Monroe ${ }^{3}$, Bruno D.

Fornage $^{4}$, Erich M. Sturgis ${ }^{4}$, J. Matthew Debnam $^{5}$

\section{Affiliations}

1 Internal Medicine, University of Oklahoma Health Sciences Center, Oklahoma City, United States

2 Pathology, University of Texas MD Anderson Cancer Center Department of Pathology, Houston, United States

3 MD Anderson Cancer Center, Radiology, Houston, United States
4 Head and Neck Surgery, Houston, University of Texas MD Anderson Cancer Center,United States

5 Radiology, University of Texas MD Anderson Cancer Center, Houston, United States

\section{Correspondence}

Dr. Ryan Bou Said

Bibliography

Internal Medicine

University of Oklahoma Health Sciences

Center

SL Young Boulevard

73104-5410

Oklahoma City

United States

Tel.: + 1/405/5939 143

ryn_293@hotmail.com
DOI https://doi.org/10.1055/a-0752-9715

Ultrasound Int Open 2018; 4: E136-E138

(c) Georg Thieme Verlag KG Stuttgart .

New York

ISSN 2199-7152

\section{(ㄷ) (2) (1)}

